Pacific Journal of Mathematics

CONJUGATIONS ON STABLY ALMOST COMPLEX 


\title{
CONJUGATIONS ON STABLY ALMOST COMPLEX MANIFOLDS
}

\author{
Allan L. EDELson
}

\begin{abstract}
A stably almost complex structure on a smooth manifold $M$ is an automorphism $J: \tau_{M} \oplus \theta^{k} \rightarrow \tau_{M} \oplus \theta^{k}$ for some $k \geqq 0$, covering the identity map on $M$, and satisfying $J^{2}=-1$. If $k=0, J$ is an almost complex structure. An involution $T: M \rightarrow M$ is a conjugation of $(M, J)$ if there exists an involution $\alpha: \theta^{k} \rightarrow \theta^{k}$ covering $T$, such that $T_{*} \oplus \alpha$ is conjugate linear, i.e., $\left(T_{*} \oplus \alpha\right) \circ J=-J \circ\left(T_{*} \oplus \alpha\right)$. The bordism theory of conjugations has been studied by $R$. Stong. In $\S 2$ of this article it is shown that every closed $n$-manifold can be realized as the fixed point set of a conjugation on a closed, $2 n$-dimensional stably almost complex manifold. This should be compared to the result of Conner and Floyd that the fixed point set of a conjugation on an almost complex $2 n$ manifold is $n$-dimensional, which is false for stably almost complex manifolds. The proof will use the following result:
\end{abstract}

LEMMA 1. Every closed manifold is cobordant to the fixed point set of a conjugation on a closed, almost complex manifold.

Let $H_{m, n}(C) \subset P^{m}(C) \times P^{n}(C)$ with $m \leqq n$, be the hypersurface defined as the locus of $w_{0} z_{0}+w_{1} z_{1}+\cdots+w_{m} z_{m}=0$ (in homogeneous coordinates $\left(w_{0}, \cdots, w_{m}\right)$ and $\left.\left(z_{0}, \cdots, z_{n}\right)\right)$. Let $H_{m, n}(R)$ be the corresponding real hypersurface. Then generators for the cobordism ring $\eta_{*}$ can be taken to be the manifolds $P^{2 n}(R)$ and $H_{m, n}(R)$, which are fixed point sets of conjugations on $P^{2 n}(C)$ and $H_{m, n}(C)$ respectively. The preceding lemma follows easily.

In $\S 3$, almost complex conjugations on $S^{2 q+1} \times S^{2 q+1}$ are given, with fixed point set $S^{2 q+1}$. As a consequence, any manifold obtained from $P^{2 n}(R)$ or $H_{m, n}(R)$ by surgeries on odd dimensional spheres, is itself the fixed point set of a conjugation on an almost complex manifold.

We will also need the following definition. If $T$ is a free involution on a compact manifold $M$, a characteristic submanifold for $(M, T)$ is a submanifold $M^{\prime} \subset M$ of codimension 1 , such that $M=$ $W_{+} \cup W_{-}$(where $W_{+}$and $W_{-}$are compact submanifolds of $M$ ), $M^{\prime}=W_{+} \cap W_{-}$, and $T\left(W_{+}\right)=W_{-} . \quad M^{\prime}$ can always be obtained as the pullback of $P^{N-1}$ by an equivariant map $(M, T) \rightarrow\left(P^{N}, A\right)$, where $A$ is the antipodal map.

2. Stably almost complex structures. 
LEMma 2. The tangent sphere bundle of a manifold is stably almost complex and the bundle involution is a conjugation.

Proof. Let $D(M)$ denote the tangent disc bundle of $M$, and $S(M)$ the sphere bundle, with projection map $\pi$. There is an isomorphism $\tau_{D(M)} \cong \pi^{*} \tau_{M} \oplus \pi^{*} \tau_{M}$, and an almost complex structure can be defined by $(x, y) \rightarrow(-y, x)$. The bundle involution acts as -1 in the bundle tangent to the fibres, identified with the second summand, and is a conjugation. Restricting to $S(M)$ gives a conjugation on $\tau_{S(M)} \oplus \nu_{S(M)}$, $\nu$ being the normal bundle to the boundary which is $\theta^{1}$.

This lemma provides an important example of stably almost complex manifolds. We are now ready to state the main result of this section.

Theorem 1. Every closed n-dimensional manifold is the fixed point set of a conjugation on a closed $2 n$-dimensional stably almost complex manifold.

Proof. Choose a cobordism $\left(W^{n+1}, F_{1}, F_{2}\right)$ with $F_{2}$ an arbitrary closed $n$-manifold. Assume $F_{1}$ is the fixed point set of a conjugation on the closed, almost complex manifold $M_{1}$. We will construct a closed, stably almost complex $2 n$-manifold $M_{2}$, with conjugation having fixed point set $F_{2}$. Let $B$ denote the tangent sphere bundle to $W$. Then $b B$ is the unit sphere bundle in $\tau_{b W} \oplus \nu_{b W}$, and the normal bundle of $b B$ in $B$ is trivial. There is then an induced stable almost complex structure and conjugation on $b B$. Note that throughout this paper, $b M$ will denote the boundary of the manifold $M$.

Lemma 3. The tangent sphere bundle to $b W$ is a stably almost complex submanifold of $b B$, invariant under the conjugation.

Proof. Over $b W$ the bundle $\tau_{W}$ splits as $\tau_{b W W} \oplus \nu_{b W}$ and $\pi^{*} \nu_{b W}$ can be identified with the normal bundle in $b B$, of the tangent sphere bundle to $b W$. This normal bundle is trivial, so there is an induced stable almost complex structure. Now let $S$ denote the tangent sphere bundle to $b W$.

LEMmA 4. There is a stably almost complex submanifold $V \subset B$, invariant under the conjugation, with $b V=V \cap b B=S$.

Proof. The involution $T$ on $B$ is free, and $S$ is a characteristic submanifold for the restriction $\left.T\right|_{b B}$. There is a map $f: b B / T \rightarrow P^{v}$, 
for $N$ sufficiently large, that is transverse regular on $P^{N-1}$ and with $S / T=f^{-1}\left(P^{N-1}\right) . \quad f$ extends to a map $F: B / T \rightarrow P^{N}$, transverse regular on $P^{N-1}$. Pulling back $P^{N-1}$ under $F$ and lifting to the twofold covering gives the desired submanifold $V$. Notice that $S$ is the disjoint union of the tangent sphere bundles to $F_{1}$ and $F_{2}$.

There is a submanifold, $V^{\prime}$, of the tangent disc bundle to $W$ consisting of $V$ and the tangent disc bundle of $b W$. This has trivial normal bundle and is invariant under $T$. There are corners along $S$, which can be rounded off preserving the triviality of the normal bundle, and we obtain a smooth, stably almost complex manifold with conjugation. The fixed point set of the conjugation is $F_{1} \cup F_{2}$.

Choose a neighborhood $N^{\prime}$ of $F_{1}$ in $V^{\prime}$, equivariantly diffeomorphic to the tangent bundle of $F_{1}$. Similarly choose a neighborhood $N$ of $F_{1}$ in $M_{1}$. Define a diffeomorphism from $N \backslash F_{1} \rightarrow N^{\prime} \backslash F_{1}$ by sending $(x, v) \rightarrow\left(x,-v /\|v\|^{2}\right)$, where $v$ is a tangent vector at $x$. This is smooth, and preserves the almost complex structure along the unit sphere bundle. Form a smooth manifold $M_{2}$ from $V^{\prime} \backslash F_{1} \cup M_{1} \backslash F_{1}$ by identifying the above submanifolds. There are almost complex structures on $V^{\prime} \backslash N_{1}^{\prime}$ and $M_{1} \backslash N_{1}$, where $N_{1}^{\prime}$ and $N_{2}$ are the vectors of length $\leqq 1$. These agree on sphere bundles, and hence $M_{2}$ has a stable almost complex structure, provided we add to $\tau_{V^{\prime}}$ a trivial complex line bundle. The involution on $M_{1} \backslash F_{1}$ is free and hence the fixed point set is $F_{2}$. This completes the proof of Theorem 1 .

3. Conjugations on $S^{2 q+1} \times S^{2 q+1}$. In [1], Calabi and Eckmann have described almost complex structures on $S^{2 q+1} \times S^{2 q+1}$. In this section we will describe a conjugation having fixed point set $S^{2 q+1}$. We begin with a description of the principal bundles involved.

Let $\left\{U_{i}\right\}_{0 \leqq i \leqq q}$ be the standard open covering of $P^{q}(C)$ by coordinate neighborhoods. Then $\left\{U_{i} \times U_{\alpha}\right\}_{0 \leqq i, \alpha \leqq q}$ is an open covering of $P^{q}(C) \times P^{q}(C)$ by coordinate neighborhoods. Let $U_{\imath \alpha}=U_{i} \times U_{\alpha}$. As in [4, Ch. 9], define a principal bundle $B$ over $P^{q}(C) \times P^{q}(C)$ with group $G=S^{1} \times S^{1}$ and transition functions $\Psi_{i \alpha, j \beta}: U_{i \alpha} \cap U_{j \beta} \rightarrow G$ given by

$$
\Psi_{i \alpha, j \beta}([Z],[W])=\left(\frac{z_{i}\left|z_{j}\right|}{z_{j}\left|z_{i}\right|}, \frac{w_{\alpha}\left|w_{\beta}\right|}{w_{\beta}\left|w_{\alpha}\right|}\right)
$$

Note that $Z=\left(z_{0}, \cdots, z_{q}\right), W=\left(w_{0}, \cdots, w_{q}\right)$, and $Z \in S^{2 q+1}, W \in S^{2 q+1}$. Then $\Psi_{i \alpha, j \beta} \Psi_{j \beta, k \gamma}=\Psi_{i \alpha, k \gamma}$. Now let $B_{i \alpha}=U_{i \alpha} \times G$ and define $\widetilde{T}_{i \alpha}: B_{i \alpha} \rightarrow$ $B_{\alpha i}$ by $\widetilde{T}_{i \alpha}([Z],[W], \lambda, \mu)=([\bar{W}],[\bar{Z}], \bar{\mu}, \bar{\lambda})$.

Lemma 5. The map $\widetilde{T}: B \rightarrow B$ defined by $\left.\widetilde{T}\right|_{B_{i \alpha}}=\widetilde{T}_{i \alpha}$ is a welldefined involution covering $T([Z],[W])=([\bar{W}],[\bar{Z}])$. 
Proof. We need to show that the diagram

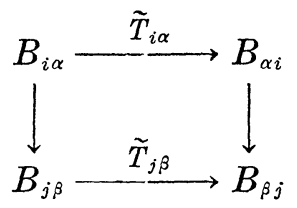

in which the vertical maps are the identifications defined on the appropriate intersections, is commutative. We have

$$
\begin{aligned}
\Psi_{\alpha i, \beta j} \widetilde{T}_{i \alpha}([Z],[W], \lambda, \mu) & =\left([W],[Z], \frac{\bar{w}_{\alpha}\left|\bar{w}_{\beta}\right|}{\bar{w}_{\beta}\left|\bar{w}_{\alpha}\right|} \bar{\mu}, \frac{\bar{z}_{i}\left|\bar{z}_{j}\right|}{\bar{z}_{j}\left|\bar{z}_{i}\right|} \bar{\lambda}\right) \\
& =\widetilde{T}_{j \beta} \Psi_{i \alpha, j \beta}([Z],[W], \lambda, \mu)
\end{aligned}
$$

and so the diagram commutes. The remainder of the lemma is clear. Note the use of the symbol $\Psi_{i \alpha, j \beta}$ to denote the map $B_{i \alpha} \rightarrow \beta_{j \alpha}$ defined on the appropriate intersection.

Define a map $h_{i \alpha}: B_{i \alpha} \rightarrow S^{2 q+1} \times S^{2 q+1}$ by

$$
h_{i \alpha}([Z],[W], \lambda, \mu)=\left(\lambda \frac{z_{i}}{\left|z_{i}\right|} Z, \mu \frac{w_{\alpha}}{\left|w_{\alpha}\right|} W\right) .
$$

Then $h_{j \beta} \Psi_{i \alpha, j \beta}=h_{i \alpha}$ so that there is a well-defined diffeomorphism $h: B \rightarrow S^{2 q+1} \times S^{2 q+1}$.

Lemma 6. The involution

$$
h \widetilde{T} h^{-1}: S^{2 q+1} \times S^{2 q+1} \longrightarrow S^{2 q+1} \times S^{2 q+1}
$$

is given by $(Z, W) \rightarrow(\bar{W}, \bar{Z})$.

Proof. We have

$$
h_{\alpha i} \widetilde{T}_{i \alpha}([Z],[W], \lambda, \mu)=\left(\bar{\mu} \frac{\bar{w}_{\alpha}}{\left|\bar{w}_{\alpha}\right|} \bar{W}, \bar{\lambda} \frac{\bar{z}_{i}}{\left|\bar{z}_{i}\right|} \bar{Z}\right),
$$

and the lemma follows.

Again following [4], consider the principal bundle $B^{\prime}$ over $P^{q}(C) \times P^{q}(C)$ with group $G^{\prime}=C / D$ where $D$ is the subgroup of $C$ generated by the complex numbers $\{1, i\}$. Define transition functions $\Psi_{i \alpha, j \beta}^{\prime}: U_{i \alpha} \cap U_{j \beta} \rightarrow G^{\prime}$ by

$$
\begin{aligned}
\Psi_{i \alpha, j \beta}^{\prime}([Z],[W])= & -\frac{1}{2 \pi i}\left(\log \left|z_{i}\right|+i \log \left|w_{\alpha}\right|\right)+\frac{1}{2 \pi i}\left(\log \frac{z_{i}}{z_{j}}+i \log \frac{w_{\alpha}}{w_{\beta}}\right) \\
& +\frac{1}{2 \pi i}\left(\log \left|z_{j}\right|+i \log \left|w_{\beta}\right|\right) .
\end{aligned}
$$


We wish to define a bundle equivalence $f: B \rightarrow B^{\prime}$. First define an isomorphism $g: G \rightarrow G^{\prime}$ by

$$
g(\lambda, \mu)=\left(\frac{1}{2 \pi i} \log \lambda\right)+i\left(\frac{1}{2 \pi i} \log \mu\right) .
$$

It follows that $g \Psi_{i \alpha j \beta}=\Psi_{i \alpha, j \beta}^{\prime}: U_{i \alpha} \cap U_{j \beta} \rightarrow G^{\prime}$, and hence that $f$ can be defined by defining $f_{i \alpha}=1 \times g: B_{i \alpha} \rightarrow B_{i \alpha}^{\prime}$. There is an induced involution $\widetilde{T}_{i \alpha}^{\prime}=(1 \times g) \widetilde{T}_{i \alpha}\left(1 \times g^{-1}\right): B_{i \alpha}^{\prime} \rightarrow B_{\alpha i}^{\prime}$ given by

$$
\widetilde{T}_{\dot{i} \alpha}^{\prime}([Z],[W],[v])=([\bar{W}],[\bar{Z}],[i \bar{v}]),
$$

and an involution $\widetilde{T}^{\prime}: B^{\prime} \rightarrow B^{\prime}$. Here $[v]$ denotes the class in $G^{\prime}$ of the complex number $v$.

Lemma 6. $\widetilde{T}^{\prime}$ is a conjugation of the complex manifold $B^{\prime}$.

Proof. In local coordinates, $\widetilde{T}^{\prime}$ is given by $\widetilde{T}^{\prime}([Z],[W],[v])=$ ([ $[\bar{W}],[\bar{Z}],[i \bar{v}])$. We need only verify that the map $[v] \rightarrow[i \bar{v}]$ is a conjugation of the complex manifold $G^{\prime}=C / D$. Since this map sends $[i v]$ to $[(-i) i \bar{v}]$, the lemma follows.

THEOREM 2. $S^{2 q+1}$ is the fixed point set of a conjugation $S^{2 q+1} \times$ $\mathrm{S}^{2 q+1}$.

Proof. The diffeomorphisms $f:(B, \widetilde{T}) \rightarrow\left(B^{\prime}, \widetilde{T}^{\prime}\right)$ and $h:(B, \widetilde{T}) \rightarrow$ $\left(S^{2 q+1} \times S^{2 q+1}, \bar{T}\right)$ are equivariant with respect to the given involutions, and commute with the projections onto $P^{q}(C) \times P^{q}(C)$. Note that $\bar{T}$ is defined by $\bar{T}(Z, W)=(\bar{W}, \bar{Z})$. Then theorem follows since the fixed point set of $\bar{T}$ is diffeomorphic to $S^{2 q+1}$.

\section{REFERENCES}

1. E. Calabi and B. Eckmann, A class of compact, complex manifolds which are not algebraic, Annals of Math., 58 (1953), 494-500.

2. P. E. Conner and E. E. Floyd, Differentiable Periodic Maps, Springer-Verlag, Berlin, 1969.

3. — Differentiable Periodic Maps, Springer-Verlag, Berlin, 1964.

4. S. Kobayashi and K. Nomizu, Foundations of Differential Geometry, vol. 2, Interscience Publishers, New York, 1969.

5. P. S. Landweber, Fixed point free conjugations on complex manifolds, Annals of Math., 86 (967), 491-502.

6. J. Milnor, Stiefel-Whitney numbers of complex manifolds and of spin manifolds, Topology v. III, (1965), 223-230.

7. R. E. Stong, Notes on Cobordism Theory, Princeton Univ. Press, 1968.

Received April 6, 1973 and in revised form August 7, 1973.

University of California, Davis 



\section{PACIFIC JOURNAL OF MATHEMATICS}

\section{EDITORS}

RICHARD ARENS (Managing Editor)

University of California

Los Angeles, California 90024

\section{J. DUGUNDJI}

Department of Mathematics University of Southern California Los Angeles, California 90007

D. Gilbarg and J. Milgram

Stanford University

Stanford, California 94305

University of Washington
Seattle, Washington 98105

ASSOCIATE EDITORS
E. F, BECKENBACH
B. H. NEUMANN
F. WOLF
K. Yoshida

\section{SUPPORTING INSTITUTIONS}

\author{
UNIVERSITY OF BRITISH COLUMBIA \\ CALIFORNIA INSTITUTE OF TECHNOLOGY \\ UNIVERSITY OF CALIFORNIA \\ MONTANA STATE UNIVERSITY \\ UNIVERSITY OF NEVADA \\ NEW MEXICO STATE UNIVERSITY \\ OREGON STATE UNIVERSITY \\ UNIVERSITY OF OREGON \\ OSAKA UNIVERSITY
}

\author{
UNIVERSITY OF SOUTHERN CALIFORNIA \\ STANFORD UNIVERSITY \\ UNIVERSITY OF TOKYO \\ UNIVERSITY OF UTAH \\ WASHINGTON STATE UNIVERSITY \\ UNIVERSITY OF WASHINGTON \\ * * * * \\ AMERICAN MATHEMATICAL SOCIETY \\ NAVAL WEAPONS CENTER
}

The Supporting Institutions listed above contribute to the cost of publication of this Journal, but they are not owners or publishers and have no responsibility for its content or policies.

Mathematical papers intended for publication in the Pacific Journal of Mathematics should be in typed form or offset-reproduced, (not dittoed), double spaced with large margins. Underline Greek letters in red, German in green, and script in blue. The first paragraph or two must be capable of being used separately as a synopsis of the entire paper. Items of the bibliography should not be cited there unless absolutely necessary, in which case they must be identified by author and Journal, rather than by item number. Manuscripts, in duplicate if possible, may be sent to any one of the four editors. Please classify according to the scheme of Math. Rev. Index to Vol. 39. All other communications to the editors should be addressed to the managing editor, or Elaine Barth, University of California, Los Angeles, California, 90024.

100 reprints are provided free for each article, only if page charges have been substantially paid. Additional copies may be obtained at cost in multiples of 50 .

The Pacific of Journal Mathematics is issued monthly as of January 1966. Regular subscription rate: $\$ 72.00$ a year (6 Vols., 12 issues). Special rate: $\$ 36.00$ a year to individual members of supporting institutions.

Subscriptions, orders for back numbers, and changes of address should be sent to Pacific Journal of Mathematics, 103 Highland Boulevard, Berkeley, California, 94708.

PUBLISHED BY PACIFIC JOURNAL OF MATHEMATICS, A NON-PROFIT CORPORATION

Printed at Kokusai Bunken Insatsusha (International Academic Printing Co., Ltd.), 270, 3-chome Totsuka-cho, Shinjuku-ku, Tokyo 160, Japan.

Copyright (C) 1973 by Pacific Journal of Mathematics Manufactured and first issued in Japan 


\section{Pacific Journal of Mathematics}

\section{Vol. 53, No. $2 \quad$ April, 1974}

Kenneth Abernethy, On characterizing certain classses of first countable spaces by

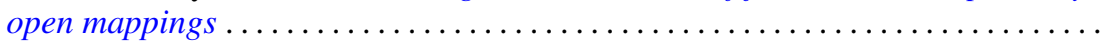

Ross A. Beaumont and Donald Lawver, Strongly semisimple abelian groups .......

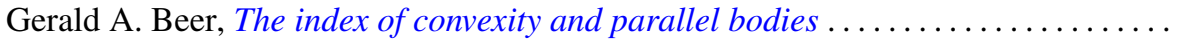

Victor P. Camillo and Kent Ralph Fuller, On Loewy length of rings ..............

Stephen LaVern Campbell, Linear operators for which $T^{*} T$ and $T T^{*}$ commute.

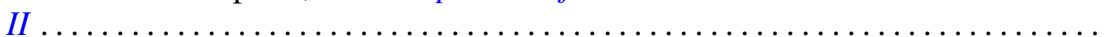

Charles Kam-Tai Chui and Philip Wesley Smith, Characterization of a function by

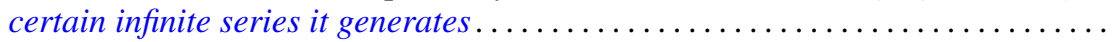

Allan L. Edelson, Conjugations on stably almost complex manifolds . ...........

Patrick John Fleury, Hollow modules and local endomorphism rings . . ..........

Jack Tilden Goodykoontz, Jr., Connectedness im kleinen and local connectedness in

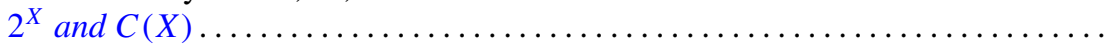

Robert Edward Jamison, II, Functional representation of algebraic intervals .......

Athanassios G. Kartsatos, Nonzero solutions to boundary value problems for

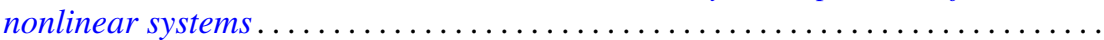

Soon-Kyu Kim, Dennis McGavran and Jingyal Pak, Torus group actions on simply

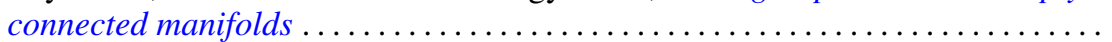

David Anthony Klarner and R. Rado, Arithmetic properties of certain recursively

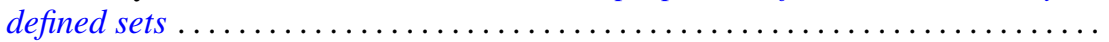

Ray Alden Kunze, On the Frobenius reciprocity theorem for square-integrable

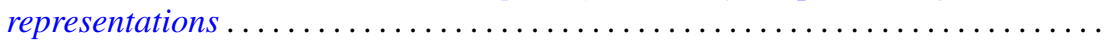

John Lagnese, Existence, uniqueness and limiting behavior of solutions of a class of differential equations in Banach space...

Teck Cheong Lim, A fixed point theorem for families on nonexpansive mappings Lewis Lum, A quasi order characterization of smooth continua

Andy R. Magid, Principal homogeneous spaces and Galois extensions . .

Charles Alan McCarthy, The norm of a certain derivation ..... . .

Louise Elizabeth Moser, On the impossibility of obtaining $S^{2} \times S^{1}$ by elementary surgery along a knot. .

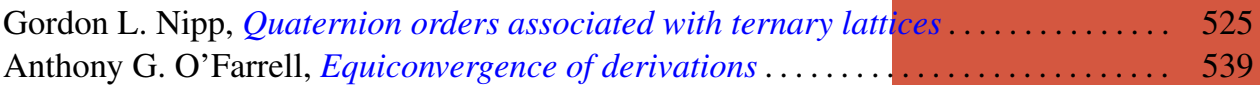

Dorte Olesen, Derivations of $A W^{*}$-algebras are inner . . . . . . . . . . . . . . . 555

Dorte Olesen and Gert Kjærgaard Pedersen, Derivations of $C^{*}$-algebras have

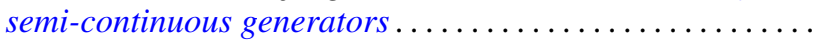

Duane O’Neill, On conjugation cobordism.

Chull Park and S. R. Paranjape, Probabilities of Wiener paths crossing differentiable

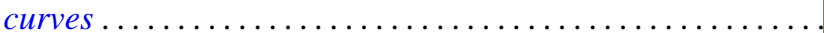

Edward Ralph Rozema, Almost Chebyshev subspaces of $L^{1}(\mu$;

Lesley Millman Sibner and Robert Jules Sibner, A note on the Atiyah-Bott fixed

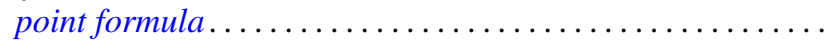

Betty Salzberg Stark, Irreducible subgroups of orthogonal groups generated by

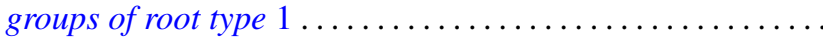

N. Stavrakas, A note on starshaped sets, $(k)$-extreme points and the half ray

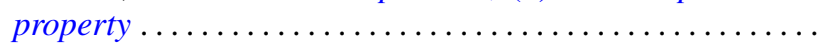

Carl E. Swenson, Direct sum subset decompositions of $Z \ldots \ldots$ 